\title{
Comparison of Chromosome Breakage in Non-Mosaic and Mosaic Patients with Fanconi Anemia, Relatives, and Patients with Other Inherited Bone Marrow Failure Syndromes
}

\author{
John H. Fargo ${ }^{a}$ b Andrzej Rochowskid ${ }^{d}$ Neelam Giri ${ }^{a}$ Sharon A. Savage ${ }^{a}$ \\ Susan B. Olson ${ }^{c}$ Blanche P. Alter ${ }^{a}$ \\ ${ }^{a}$ Clinical Genetics Branch, Division of Cancer Epidemiology and Genetics, National Cancer Institute, National Institutes \\ of Health, Department of Health and Human Services, Bethesda, Md., ${ }^{b}$ Center for Cancer and Blood Disorders, \\ Children's National Medical Center, Washington, D.C., and ' Department of Molecular and Medical Genetics, Oregon \\ Health \& Science University, Portland, Oreg., USA; ${ }^{\text {d } Q u e e n s w a y ~ C a r l e t o n ~ H o s p i t a l, ~ O t t a w a, ~ O n t ., ~ C a n a d a ~}$
}

\section{Key Words}

Chromosome breakage · Diepoxybutane · Fanconi anemia · Inherited bone marrow failure $\cdot$ Mitomycin C

\begin{abstract}
Fanconi anemia $(F A)$ is a rare inherited bone marrow failure syndrome (IBMFS). Affected individuals must be distinguished from relatives, patients with mosaicism must be identified, and patients with other IBMFS classified as non$F A$. The diagnostic feature of FA is increased chromosomal breakage in blood lymphocytes cultured with diepoxybutane or mitomycin C. Here, we sought a method to uniquely identify patients with FA with mosaicism, using cells from participants in the National Cancer Institute IBMFS cohort. Lymphocytes were treated with diepoxybutane or mitomycin C, and metaphases scored for breaks and radials. Analyses included the percentage of cells with any aberration, breaks per cell, and breaks per aberrant cell. There were 26 patients with FA (4 mosaics), 46 FA relatives, and 62 patients with a non-FA IBMFS. By all analytic methods, patients with
\end{abstract}

FA were abnormal compared with other groups. Those with FA mosaicism had more breakage than relatives or patients with non-FA IBMFS, but there was some individual overlap. The choices of clastogen are laboratory-dependent, but there was no method or analysis of lymphocytes that clearly distinguished all individuals mosaic for FA from relatives or patients with other IBMFS. Thus, genotyping remains the best method for providing absolute clarity.

(c) 2014 S. Karger AG, Basel

Fanconi anemia (FA, OMIM 227650) is a rare inherited bone marrow failure syndrome (IBMFS) characterized by pancytopenia, congenital malformations, and a high risk of cancer [Alter et al., 2010; Shimamura and Alter, 2010]. It is phenotypically and genetically heterogeneous, with at least 16 genes whose products cooperate in the FA/BRCA DNA repair pathway [Bogliolo et al., 2013]. The inheritance is autosomal recessive, except FANCB which is X-linked recessive. Increased chromosome breakage is the diagnostic feature of FA, due to ge-

\section{KARGER}

E-Mail karger@karger.com

www.karger.com/cgr
(C) 2014 S. Karger AG, Basel

$1424-8581 / 14 / 1441-0015 \$ 39.50 / 0$ 
nomic instability. Schroeder et al. [1964] were the first to observe increased chromosome breakage in cultures of lymphocytes from patients with FA compared with normal cultures. Subsequently, Schuler et al. [1969] reported that addition of DNA cross-linking agents to the cultures consistently led to increased breakage rates in FA. Agents with similar effects include nitrogen mustard, cyclophosphamide and ionizing irradiation [Higurashi and Conen, 1971; Sasaki and Tonomura, 1973; Auerbach and Wolman, 1976; Berger et al., 1980a, b; Deviren et al., 2003]. However, most centers currently use diepoxybutane (DEB) or mitomycin C (MMC) [Auerbach et al., 1981; Cervenka et al., 1981]. There are several categories of individuals for whom the diagnosis of FA needs to be proven or ruled out, including immediate relatives (who may be carriers, have FA, or be unaffected), and patients with similar phenotypes such as bone marrow failure and/or physical findings. The currently published chromosomal breakage analyses do not appear to completely distinguish these categories.

The carrier frequency of FA in the general population is around 1 in 181 [Rosenberg et al., 2011]. Distinguishing heterozygous carriers of a mutated FA allele from those affected with FA and from non-carriers has important genetic and family planning implications. It is also important in order to understand whether FA heterozygotes have an increased cancer risk [Berwick et al., 2007]. Patients with biallelic mutations in FANCD1/ $B R C A 2$ comprise a rare FA genotype [Alter et al., 2007], and carriers of heterozygous mutations in BRCA2 have an increased risk of breast and ovarian cancer [Chen and Parmigiani, 2007]. Although modern methods for genotyping patients with FA usually lead to clear classification of the mutation status of FA relatives, the data are often not immediately available, and genetic counseling of families may precede the availability of that information.

Patients who have FA with hematopoietic somatic mosaicism are often difficult to identify both clinically and diagnostically, although it was suggested that they may comprise up to $25 \%$ of patients with FA [Lo Ten Foe et al., 1997]. The somatic molecular events underlying mosaicism in patients with FA include back mutation, intragenic crossover, gene conversion or compensating deletions/ insertions, leading to correction of one of the mutated FA alleles in an hematopoietic stem cell or lymphocyte progenitor [Kwee et al., 1983; Lo Ten Foe et al., 1997; Gregory et al., 2001; Gross et al., 2002; Alter et al., 2005; Soulier et al., 2005]. This gene reversion results in acquired heterozygosity in stem cells and their progeny, such as lymphocytes, rendering them less sensitive to the clastogenic effects of DNA cross-linking agents. However, there is no standard degree of chromosomal breakage in peripheral blood lymphocytes that clearly differentiates patients with FA with mosaicism either from FA heterozygotes or from healthy individuals without FA. If FA is highly suspected, skin fibroblasts must be analyzed despite an apparently normal chromosomal breakage result in blood lymphocytes.

Other IBMFS, such as Diamond-Blackfan anemia (DBA), dyskeratosis congenita (DC), and ShwachmanDiamond syndrome (SDS), may share some clinical and laboratory features with FA. These include physical anomalies, as well as single or multiple cytopenias, macrocytic red blood cells and elevated fetal hemoglobin levels. These syndromes also are genetically heterogeneous [Shimamura and Alter, 2010]. Distinguishing patients with these IBMFS from those with FA has genetic, therapeutic and prognostic implications. These patients might be misclassified as FA if their lymphocytes have elevated chromosomal breakage, as has been suggested previously [Dokal and Luzzatto, 1994].

Chromosomal aberrations have been analyzed in many ways, including the number of chromosomal breaks and/or radial figures per cell, percentage of cells with aberrations, and breaks or radials per aberrant cell. The clastogenic agents and the concentrations employed vary among centers. The level of abnormality used to identify patients with FA is dependent on the specific laboratory. In this study, we explored several ways of expressing breakage results, and compared data using DEB with $\mathrm{MMC}$ as the clastogen. We included representatives from all of the relevant FA groups: genetically-confirmed patients with FA without or with mosaicism, and relatives of patients with FA, as well as patients with the major non-FA IBMFS: DBA, DC, and SDS.

\section{Materials and Methods}

Participants were enrolled in the National Cancer Institute Inherited Bone Marrow Failure Syndromes protocol, NCI 02-C0052 (clinical trials.gov NCT00027274; www.marrowfailure.cancer.gov). This is an open, retrospective/prospective cohort, established in January 2002, with approval from the NCI Institutional Review Board. Data reported here are derived from individuals enrolled before November 2009. All participants or their guardians provided written informed consent in accordance with Health and Human Services regulation 45 CFR 46.

\section{Participant Classification}

Individuals with FA had many typical physical abnormalities, including skin café-au-lait spots, hyperpigmentation, short stat-
16

Cytogenet Genome Res 2014;144:15-27 DOI: $10.1159 / 000366251$
Fargo/Rochowski/Giri/Savage/Olson/ Alter 
ure, abnormal thumbs and radii, microcephaly, small eyes, abnormal ears or hearing, and abnormal kidneys, as well as varying degrees of bone marrow failure and personal or family histories of malignancies consistent with FA. Patients without physical findings who had bone marrow failure or an FA-associated malignancy were suspected if they had increased chromosome breakage. Mosaicism was considered in individuals for whom there was a high clinical index of suspicion for FA, who did not have lymphocyte chromosome breakage that met the diagnostic range of the testing laboratory, in whom skin fibroblast chromosomal breakage was diagnostic. One of the mosaic patients had a history of toxicity from radiation therapy for head and neck squamous cell carcinoma, 2 were monozygotic twins of whom one had an absent thumb and radius, and one was an adult who was a potential stem cell transplant donor for a sister with aplastic anemia [Alter et al., 2005; Mankad et al., 2006].

Biallelic mutated genes were identified in all the patients with FA, including those with mosaicism (biallelic in fibroblast DNA, heterozygous in lymphocyte DNA). Carriers of FA had a single allelic mutation in an FA gene, or were obligate carriers based on pedigree when material was not available for genotyping; these included parents and siblings. Non-carrier relatives were siblings who did not have any signs of FA, and did not have a mutation in the FA-associated gene in their family (or were genotype unknown due to lack of material). Patients with another IBMFS were categorized according to criteria for their specific IBMFS [Shimamura and Alter, 2010]. Mutations in genes for FA, DBA, DC, and SDS were identified or confirmed by bidirectional sequencing of PCRamplified fragments (GeneDx, Gaithersburg, Md., USA, or Ambry Genetics, Viejo, Calif., USA).

\section{Clastogen Treatment}

Blood was collected in sodium heparin. Depending on the white blood cell count, either whole blood, 50:50 cells:serum (if white blood cell count was low), or buffy coat (if white blood cell count was very low) were cultured in centrifuge tubes containing RPMI medium supplemented with fetal bovine serum, L-glutamine and gentamycin. Both DEB and MMC breakage analyses were conducted for each patient. DEB or MMC was added to separate cultures to achieve respective final concentrations of $100 \mathrm{ng} /$ $\mathrm{ml}$ or $40 \mathrm{ng} / \mathrm{ml}$, using methods adapted from those described [Auerbach et al., 1981; Cervenka et al., 1981]. DEB was added at $24 \mathrm{~h}$ or MMC at time zero. All cultures were harvested at $96 \mathrm{~h}$ to insure an adequate number of metaphases. Colcemid was added to each culture $1 \mathrm{~h}$ prior to harvest, at a final concentration of $0.04 \mu \mathrm{g} / \mathrm{ml}$. Cells in suspension were dropped on microscope slides, and the preparations were stained with Wright stain. Fifty cells from each clastogen were scored for chromosome breaks and radial formations using a Nikon Eclipse E800 photoscope. Representative images were captured using either CytoVision (Applied Imaging) or Applied Spectral Imaging software. Aberrations were recorded as the number of cells with specific numbers of breaks or radials, as well as the number of breaks in radial-containing cells. While the actual structure of radial figures remains unknown, each radial figure was assigned a value of 2 breaks for the purpose of incorporating these data into the overall averages. Several comparison groups were examined: total breaks per cell, percentage of aberrant cells, and breaks per aberrant cell, as well as the chromosome fragility index (CFI), which is breaks per aberrant cell multiplied by the percentage of aberrant cells [Castella et al., 2011].

Chromosome Breakage in Fanconi Anemia

\section{Statistics}

Analyses were performed using Microsoft Excel (Microsoft Office Excel 2007, Santa Rosa, Calif., USA) and Stata 13.1 (StataCorp Release 13, College Station, Tex., USA). p values $\leq 0.05$ were considered statistically significant.

\section{Results}

There were 26 patients with FA, of whom 22 were nonmosaics and 4 had hematopoietic somatic mosaicism [Alter et al., 2005; Mankad et al., 2006] (table 1). All patients with FA had mutations in known FA genes: 18 FANCA, 3 FANCC, 1 each FANCD2, FANCF, and FANCI, and 2 $F A N C J$. The 4 patients with mosaicism had documented FANCA gene correction in blood cells and biallelic mutations in skin fibroblasts. The relatives included 40 carriers of FA, 4 proven non-carriers (siblings without mutations in genes for FA), and 2 siblings where the genetic status was unknown. Six of the 40 carriers were categorized based on pedigree (parents or children of patients with FA), while 34 were proven to have a single FA mutation. The patients with other IBMFS included 31 with DC, 24 with DBA, and 7 with SDS. There were significantly more females than males among the patients with FA and more males than females among all the patients with other IBMFS, particularly the patients with DC. All relatives of patients with FA were significantly older than all patients with FA.

\section{Analyses of Groups: Patients with FA and Relatives}

As a group, the patients with FA were clearly distinguished from their relatives in the analyses of DEB cultures by the percentage of aberrant cells (cells with breaks and/or radials), breaks per cell, breaks per aberrant cell, and CFI (fig. 1A, panels a-d; table 2). The 4 patients with FA and mosaicism did not overlap with the non-mosaic patients with FA in the percentage of aberrant cells ( $p<$ 0.001 ) (fig. 1A, panel a). The mosaics were also lower than the non-mosaics in breaks per cell ( $p=0.008$ ) (fig. $1 \mathrm{~A}$, panel b), but the highest result in the mosaics was the same as the lowest in the non-mosaics, 0.6 breaks per cell. The number of breaks per aberrant cell was statistically lower in the mosaics than in the non-mosaics $(p=0.04)$ (fig. $1 \mathrm{~A}$, panel c), but there was obvious overlap. The CFI (fig. 1A, panel $d$ ) was lower in the mosaics than the non-mosaics $(\mathrm{p}<0.001)$. Thus, patients with FA and mosaicism might be distinguished from patients with FA without mosaicism based on very low percentage of aberrant cells, breaks per cell, and CFI, but not with breaks per aberrant cell, in which there was a small amount of overlap. 
One of the challenges in evaluation of families with FA is proper classification of their relatives. Carrier and noncarrier relatives were not distinguishable from each other by any method of analysis of chromosome breakage. As a group, the relatives had a significantly lower percentage of aberrant cells, fewer breaks per cell, breaks per aberrant cell, and lower CFI when compared with the entire group with FA ( $\mathrm{p}<0.001)$. However, there was overlap with the mosaic patients with FA, particularly in the breaks per aberrant cell $(\mathrm{p}=0.6)$. Thus, relatives of patients with FA were clearly distinguishable from the non-mosaic patients with FA ( $\mathrm{p}<0.001$ in all analyses), but showed some overlap with the patients with FA who were mosaics.

\section{Analyses of Groups: Patients with FA and with Other IBMFS}

Similar analyses were performed to compare the patients with FA with patients with the other IBMFS (fig. 1A, panels $\mathrm{e}-\mathrm{h}$ ). The patients with DBA and DC had significantly lower percentages of aberrant cells and fewer breaks per cell than the patients with FA and mosaicism $(\mathrm{p}<0.001)$, while the values in the patients with SDS were not significantly less than in those with FA $(\mathrm{p}=0.01)$. Breaks per aberrant cell were lowest in patients with DC $(\mathrm{p}<0.01)$, less in SDS than in mosaics with FA $(\mathrm{p}=0.04)$ and similar in mosaic patients with FA and in those with DBA $(p=0.2)$. However, since the percentage of aberrant cells and the numbers of breaks per cell were both low in the patients with non-FA IBMFS, the number of patients with DBA, DC or SDS in whom breaks per aberrant cell could be determined was very small. The CFI did distinguish patients with FA from those with DBA or DC ( $\mathrm{p}<$ $0.001)$, except for SDS ( $\mathrm{p}=0.1$ ).

Data from cultures with MMC were analyzed in a similar manner (fig. 1B, panels a-h). As with DEB, patients with FA and mosaicism were distinct from those with FA without mosaicism in all analyses $(\mathrm{p}<0.001)$ (fig. $1 \mathrm{~B}$, panels $\mathrm{a}-\mathrm{d}$ ). The mosaic patients with FA were also distinct from the relatives of all patients with FA $(\mathrm{p}<0.001)$ (fig. 1B, panels a-d). However, there was a slight overlap of the results in the other IBMFS with those in FA mosaics, in all parameters and with either DEB or MMC. These data suggest that some patients with other IBMFS may have breakage results that overlap within the range seen in patients with FA mosaicism.

The percentage of aberrant cells, numbers of breaks per cell and breaks per aberrant cells, and CFI values were higher with MMC than with $\mathrm{DEB}$, since there were more radials in the MMC cultures (fig. 1B). Radial figures were conservatively considered to contribute 2 breaks per ra-

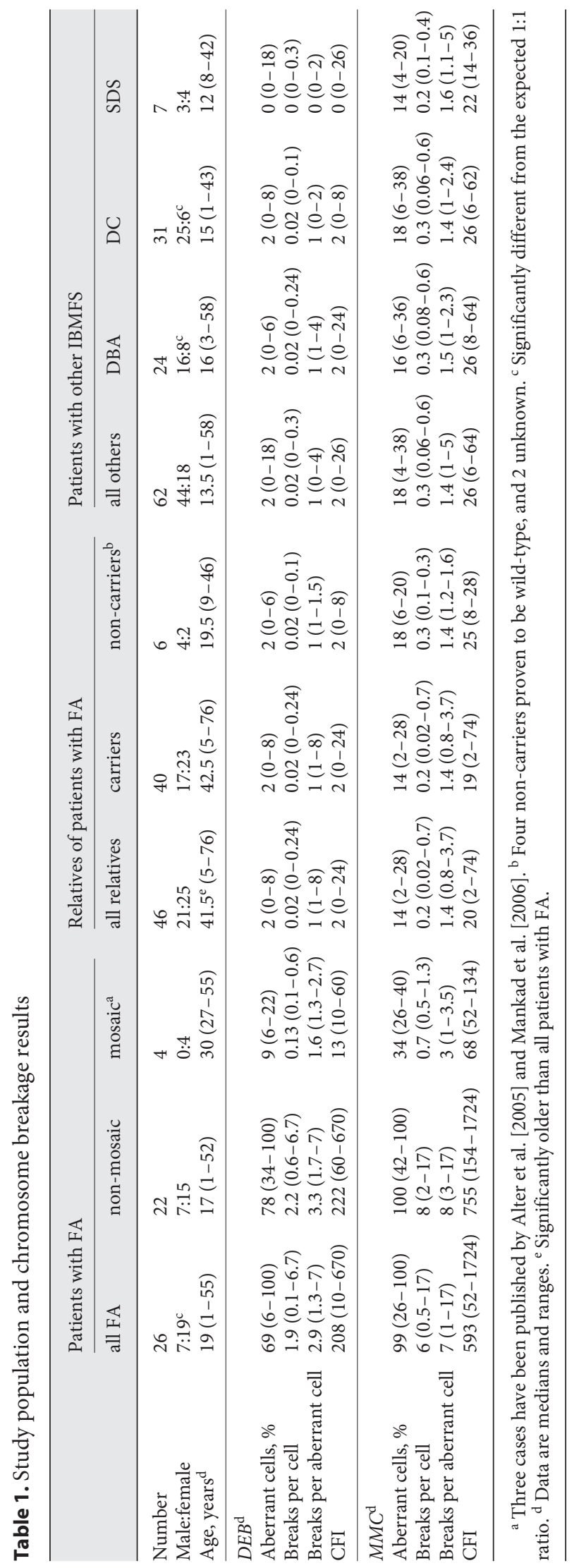

Fargo/Rochowski/Giri/Savage/Olson/ Alter 
Fig. 1. Comparison of chromosome breakage results among patients with FA, their relatives, and patients with other IBMFS. Data are shown as box and whisker plots. A DEB treatment of cultures. Percentage of aberrant cells (a, e), total breaks per cell (b, f), total breaks per aberrant cell (c, g), and CFI (\% aberrant cells $\times$ breaks per aberrant cell) $(\mathbf{d}, \mathbf{h})$ in patients with FA (All FA, $\mathrm{n}=$ 26), non-mosaic patients ( $\mathrm{NM}, \mathrm{n}=22$ ), mosaic patients $(M, n=4)$, relatives of patients with FA (All $\mathrm{R}, \mathrm{n}=46$ ), carrier relatives $(C R, n=40)$, non-carrier relatives (NCR, $\mathrm{n}=6$ ), Diamond-Blackfan anemia (DBA, $n=24)$, dyskeratosis congenita (DC, $\mathrm{n}=31$ ), and Shwachman-Diamond syndrome (SDS, $\mathrm{n}=7$ ). Mosaic patients had significantly lower percent aberrant cells, breaks per cell, and CFI than non-mosaic patients (all $\mathrm{p}<0.01$ ), and respectively higher than in relatives in the same analyses (all p $<0.001$ ). However, while the breaks per aberrant cell in the mosaic samples were lower than in the non-mosaics $(p=0.04)$, the mosaics were in the same range as the relatives $(p=0.6)$. Compared with patients with other IBMFS, the 4 mosaic patients with FA had a wider range of results. The percent aberrant cells, breaks per cell, and CFI in the mosaics were higher than in DBA and DC $(\mathrm{p}<0.001)$ but not higher than in SDS $(\mathrm{p}=0.1)$. The breaks per aberrant cells in the mosaics were similar to those in DBA. B MMC treatment of cultures. Same panels and groups as in $\mathbf{A}$. The mosaic patient samples were lower than the non-mosaics in all categories, and higher than the relatives (a-d). The mosaic patients with FA were also higher than the other IBMFS ( $\mathrm{p}<0.001)(\mathbf{e}-\mathbf{h})$, except the breaks per aberrant cell in SDS were within the range of the FA mosaics $(p=0.6)$.


Chromosome Breakage in Fanconi Anemia
Cytogenet Genome Res 2014;144:15-27 DOI: $10.1159 / 000366251$ 
Table 2. Identification of patients with FA compared with their relatives and with patients with other IBMFS

A Comparison with relatives

\begin{tabular}{lllllrrrrr}
\hline Clastogen & Analysis & $\begin{array}{l}\text { FA meeting } \\
\text { criteria }\end{array}$ & $\begin{array}{l}\text { All relatives } \\
\text { meeting criteria }\end{array}$ & $\begin{array}{l}\text { OR in favor } \\
\text { of FA }\end{array}$ & Sensitivity, \% & Specificity, \% & ROC & PPV, \% & NPV, \% \\
\hline DEB & aberrant cells $>8 \%$ & $24 / 26$ & $0 / 46$ & inf & 92 & 100 & 0.96 & 100 & 96 \\
& breaks/cell $>0.1$ & $24 / 26$ & $3 / 46$ & 172 & 92 & 94 & 0.93 & 89 & 96 \\
& breaks/aberrant cell $>1.25$ & $25 / 26$ & $10 / 46$ & 90 & 96 & 78 & 0.87 & 71 & 97 \\
& CFI $>40$ & $23 / 26$ & $0 / 46$ & inf & 89 & 100 & 0.94 & 100 & 94 \\
\hline MMC $^{\text {a }}$ & aberrant cells $>36 \%$ & $24 / 26$ & $0 / 46$ & inf & 92 & 100 & 0.96 & 100 & 96 \\
& breaks/cell $>0.56$ & $24 / 26$ & $1 / 46$ & 540 & 92 & 98 & 0.95 & 96 & 95 \\
& breaks/aberrant cell $>1.56$ & $25 / 26$ & $14 / 46$ & 57 & 96 & 70 & 0.83 & 64 & 97 \\
\hline
\end{tabular}

B Comparison with patients with other IBMFS

\begin{tabular}{|c|c|c|c|c|c|c|c|c|c|}
\hline Clastogen & Analysis & $\begin{array}{l}\text { FA meeting } \\
\text { criteria }\end{array}$ & $\begin{array}{l}\text { All other patients } \\
\text { meeting criteria }\end{array}$ & $\begin{array}{l}\text { OR in favor } \\
\text { of FA }\end{array}$ & Sensitivity, \% & Specificity, \% & ROC & PPV, \% & NPV, \% \\
\hline \multirow[t]{4}{*}{ DEB } & aberrant cells $>8 \%$ & $24 / 26$ & $1 / 62$ & 744 & 92 & 98 & 0.95 & 96 & 97 \\
\hline & breaks/cell >0.1 & $24 / 26$ & $2 / 62$ & 360 & 92 & 97 & 0.95 & 92 & 97 \\
\hline & breaks/aberrant cell $>1.25$ & $25 / 26$ & $7 / 62$ & 196 & 96 & 89 & 0.92 & 78 & 98 \\
\hline & $\mathrm{CFI}>40$ & $23 / 26$ & $0 / 62$ & $\inf$ & 89 & 100 & 0.94 & 100 & 95 \\
\hline \multirow[t]{3}{*}{$\mathrm{MMC}^{\mathrm{a}}$} & aberrant cells $>36 \%$ & $24 / 26$ & $1 / 62$ & 732 & 92 & 98 & 0.95 & 96 & 97 \\
\hline & breaks/cell $>0.56$ & $24 / 26$ & $4 / 62$ & 174 & 92 & 94 & 0.93 & 86 & 97 \\
\hline & breaks/aberrant cell $>1.56$ & $25 / 26$ & $22 / 62$ & 45 & 96 & 65 & 0.80 & 53 & 98 \\
\hline
\end{tabular}

Inf = Infinity; NPV = negative predictive value; $\mathrm{OR}=$ odds ratio; $\mathrm{PPV}=$ positive predictive value; $\mathrm{ROC}=$ area under the curve in receiver operating characteristic analysis. Subjects were identified as consistent with FA if their values met the criteria shown in the table. These cut-points are those used in the diagnostic laboratory of Dr. Susan Olson.

${ }^{\text {a }}$ CFI not available.

dial, and were then added to the breaks that were not from radials. As in the DEB cultures, the results in MMC showed that patients with FA and mosaicism had lower breakage rates than patients with FA without mosaicism ( $p<0.01$ ), but there was still a slight overlap between the results in relatives of patients with FA and mosaic patients.

The group analyses indicated that patients with FA and mosaicism were not clearly distinguished from their relatives, although their percentage of aberrant cells, breaks per cell, or CFI, with DEB or MMC, were at the low end of the range for patients with FA. Relatives had chromosome breakage that was generally lower than in patients with FA, but did overlap slightly, particularly with the data from the mosaic patients. The most helpful data were the breaks per aberrant cell, where the mosaic results were within the range of the non-mosaic patients with FA, while the values for the relatives tended to be lower than for the mosaic patients with FA. In order to explore these differences more clearly, we examined the frequency of all results on an individual basis.

\section{Analyses of Individuals with FA and Relatives or \\ Patients with Other IBMFS}

The data in figure $1 \mathrm{~A}$ and $\mathrm{B}$ are summary data comparing the different groups of participants; examination of individual chromosome breakage data appeared to be more informative (figs. 2-6). Among the patients with FA, the 4 with the lowest percentage of cells with aberrations in DEB or MMC were the mosaics (fig. 2A, D). However, several of the relatives and the patients with other IBMFS had percentages of cells with aberrations that overlapped in both DEB and MMC with 2 of the 4 mosaics (fig. 2B, C, E, F).

The breaks per cell in the mosaics were lower than in the non-mosaics (fig. 3), but most of the relatives and the other IBMFS patients overlapped with the mosaics; all 


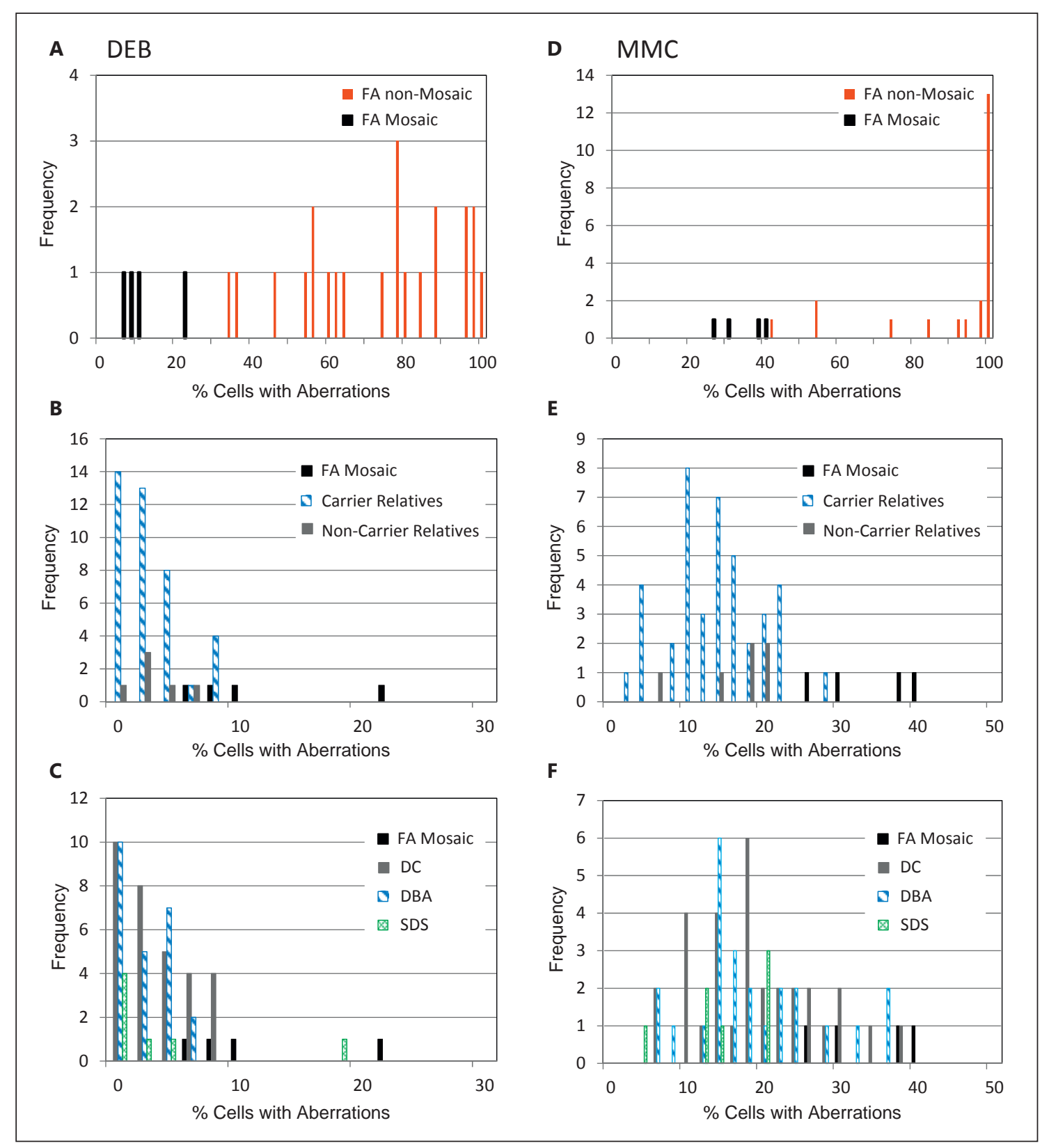

Fig. 2. Percentage of cells with aberrations per individual in cultures treated with DEB (A-C) and MMC (D-F). A, D Comparison of patients with non-mosaic FA (red bars) with patients with mosaic FA (black bars). B, E Comparison of patients with mosaic FA (black bars) with relatives of patients with FA (blue-striped bars = FA gene carrier relatives; grey bars = non-carrier relatives). C, F Comparison of patients with mosaic FA (black bars) with pa- tients with other IBMFS (grey bars = dyskeratosis congenita; bluestriped bars = Diamond-Blackfan anemia; green-stippled bars = Shwachman-Diamond syndrome). The patients with FA and mosaicism have a lower percentage of cells with aberrations than patients with FA who do not have mosaicism. However, the mosaic patients with FA overlap with relatives of FA patients, and with patients with DBA and DC. See table 2 for statistical analyses. were $\leq 1$ break per cell in DEB and slightly higher $(<2$ breaks per cell) in MMC. The breaks per aberrant cell for the mosaics were within the range seen in patients with FA, albeit at the lower end, as were the relatives and the patients with other IBMFS who had breaks (although some had none) (fig. 4).

Our data so far indicated that patients with FA and mosaicism might be distinguishable from the non-mo- 


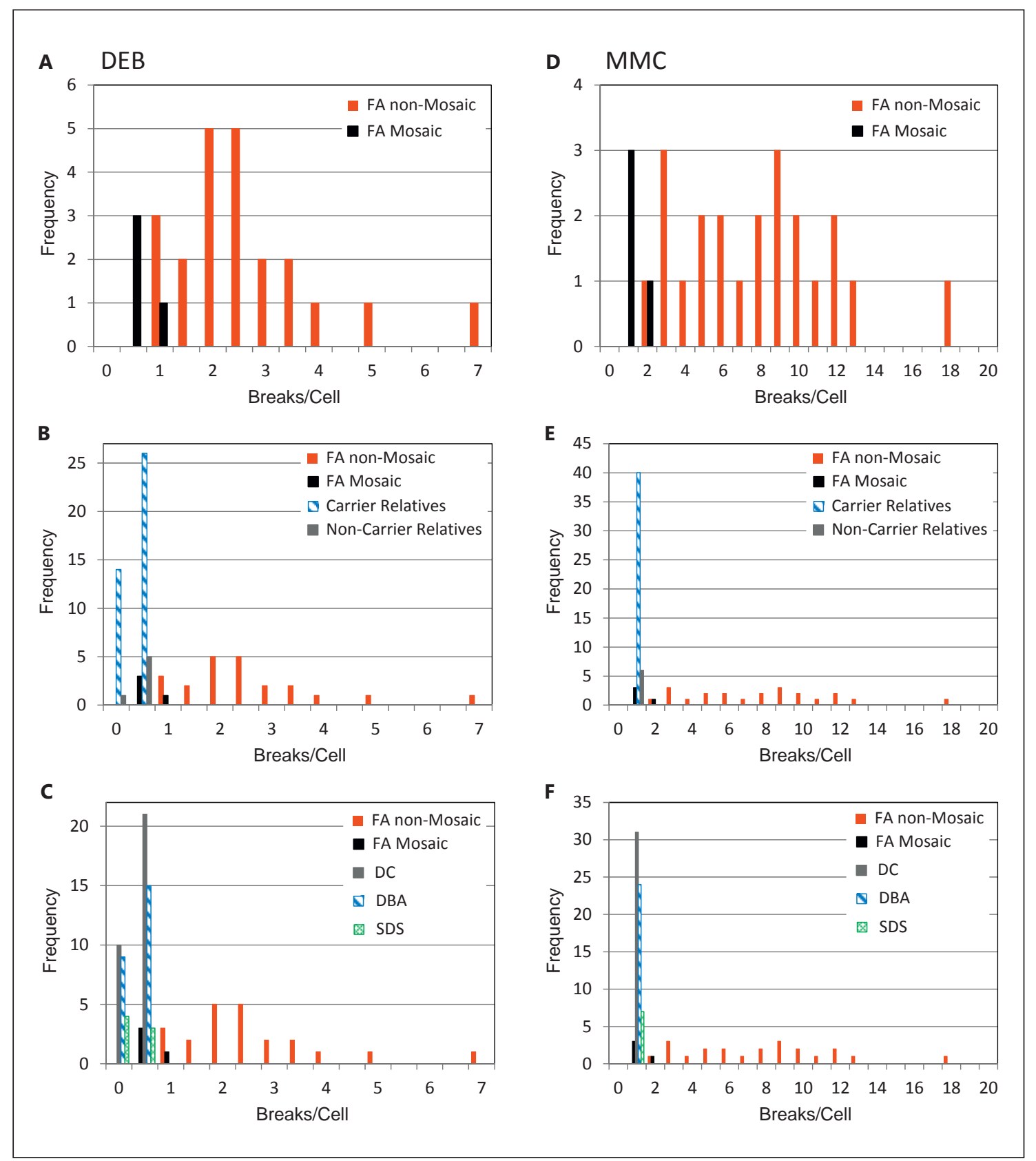

Fig. 3. Breaks per cell after DEB (A-C) and MMC (D-F) treatment of cultures. A, D Comparison of patients with non-mosaic FA with patients with mosaic FA. B, E Comparison of patients with mosaic FA with carrier and non-carrier relatives. C, F Comparison of pa- tients with mosaic FA with patients with DC, DBA, and SDS. Symbols as in figure 2. There is slight overlap between the results in patients with FA with and without mosaicism, as well as relatives and other IBMFS. saic population with FA by the presence of a lower percentage of aberrant cells using either DEB or MMC. To explore this further, we evaluated the breaks per cell versus the percentage of cells with aberrations (fig. 5). Although the data from the 4 patients with FA and mo- saicism were clearly distinct from those of the non-mosaic patients with FA, some of the relatives and the patients with other IBMFS still overlapped with the mosaic data. 


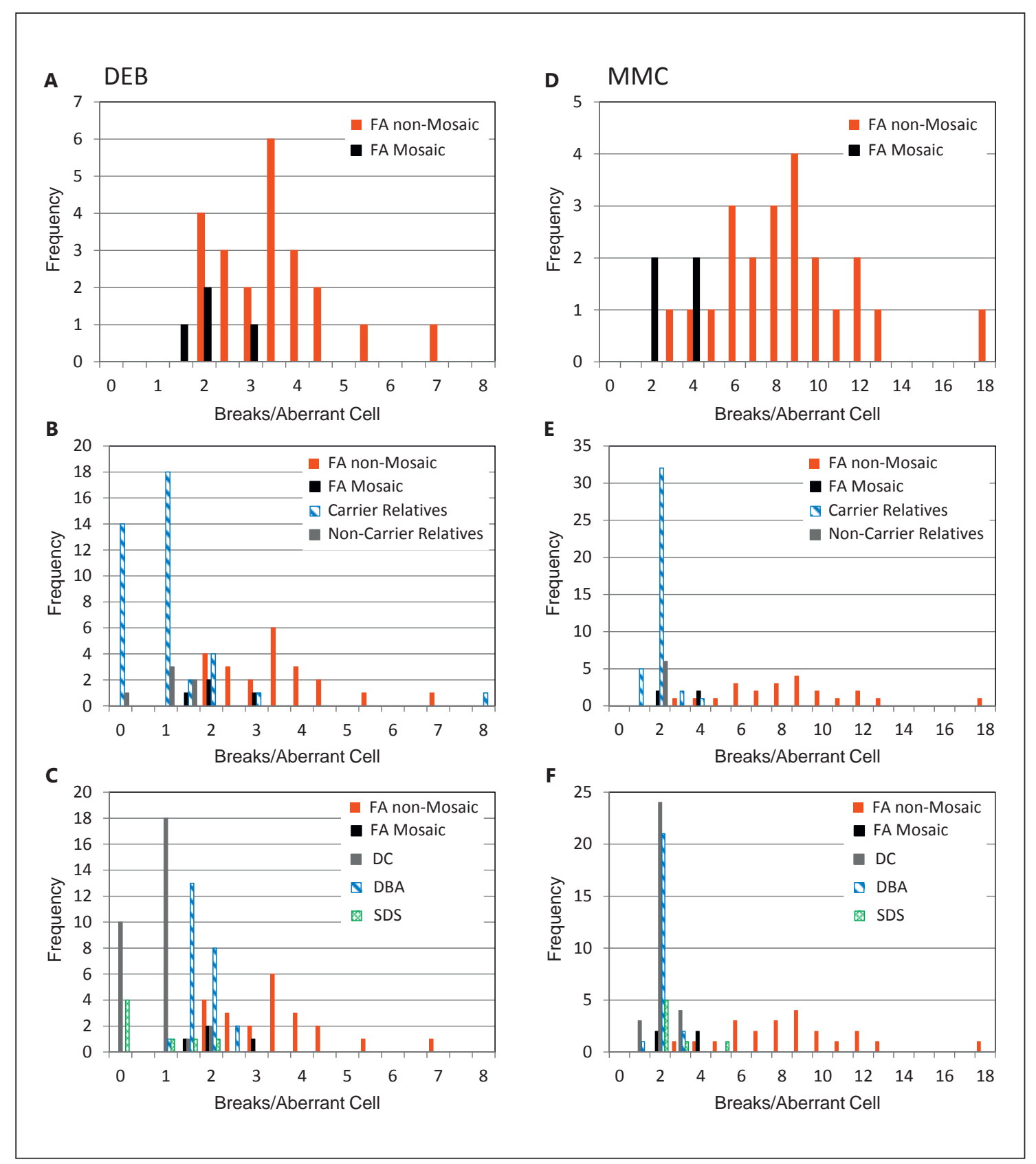

Fig. 4. Breaks per aberrant cell after DEB (A-C) and MMC (D-F) treatment of cultures. A, D Comparison of patients with non-mosaic FA with patients with mosaic FA. B, E Comparison of patients with mosaic FA with carrier and non-carrier relatives. C, F Com- parison of patients with mosaic FA with patients with other IBMFS. Symbols as in figure 2. There is overlap between the results in patients with FA with and without mosaicism, as well as relatives and other IBMFS.
We then calculated the CFI described by Castella et al. [2011]. In contrast to their clear distinction of relatives from mosaics with FA and from non-mosaics with FA at a value of 40 , we still had slight overlap of mosaics with non-mosaics, relatives with mosaics, and other IBMFS with mosaics, with either DEB or MMC (fig. 6). We were unable to identify a specific cut point using this method to analyze our own data. Over all, we did not determine a breakage analysis method that clearly distinguished all of our subject categories from each other. 


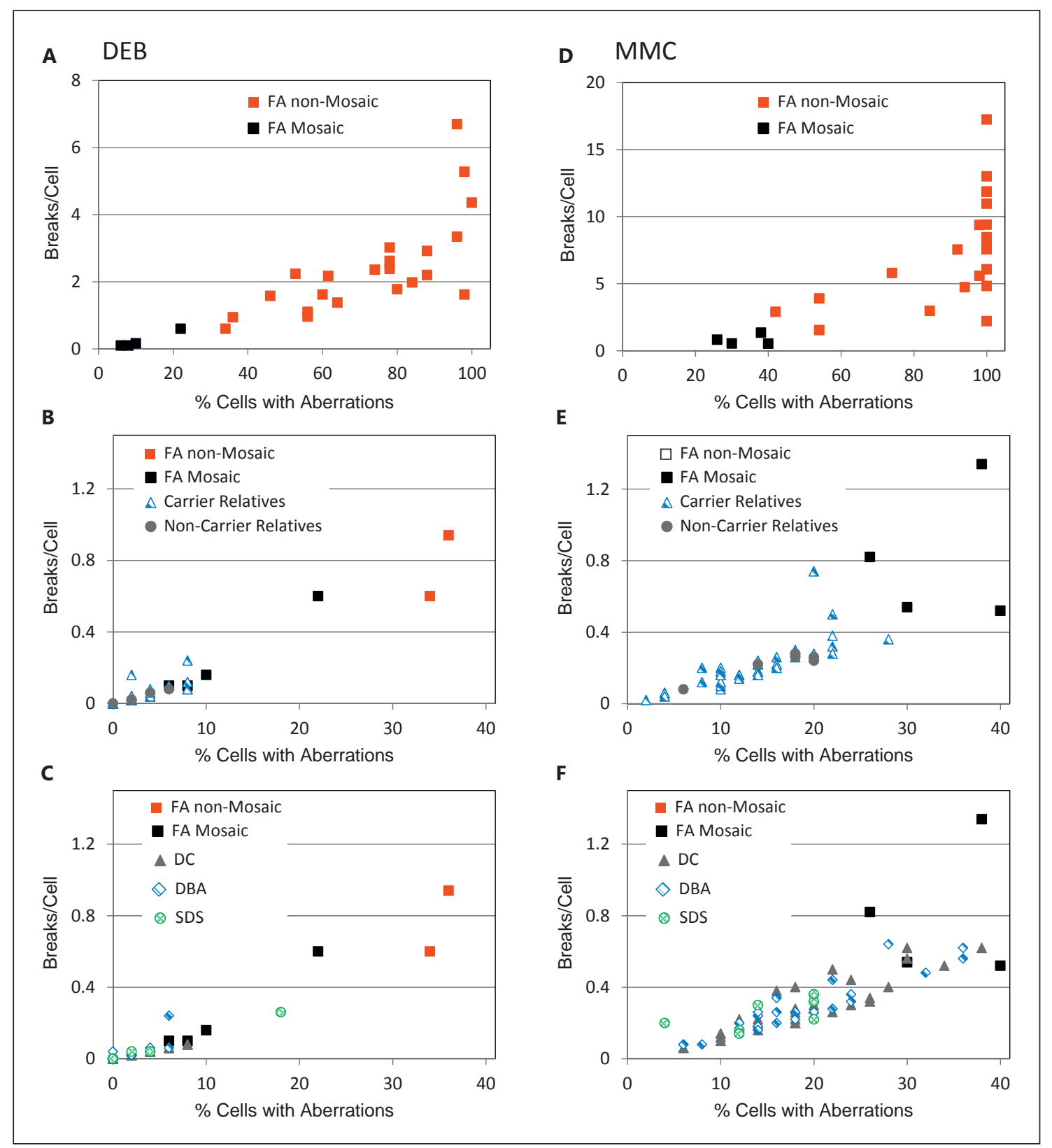

Fig. 5. Breaks per cell in relation to the percentage of aberrant cells after DEB (A-C) and MMC (D-F) treatment of cultures. A, D Comparison of patients with non-mosaic FA with patients with mosaic FA. B, E Comparison of patients with mosaic FA with relatives.
C, F Comparison of patients with mosaic FA with patients with other IBMFS. Symbols as in figure 2. The patients with mosaic FA have lower values than the patients with non-mosaic FA, but overlap with relatives and other IBMFS.

\section{Discussion}

The suspected diagnosis of FA has been confirmed for more than 30 years by analyses of chromosome aberrations in Tlymphocytes cultured with clastogenic agents. This assay is highly successful in distinguishing patients with FA from those with acquired aplastic anemia, and/or with specific congenital anomalies also seen in FA. The recognition of patients with somatic hematopoietic mosaicism has been more challenging, since a subset of their peripheral blood cells has undergone a molecular event in which $1 \mathrm{FA}$ allele reverted to normal, while the other remained mu- 


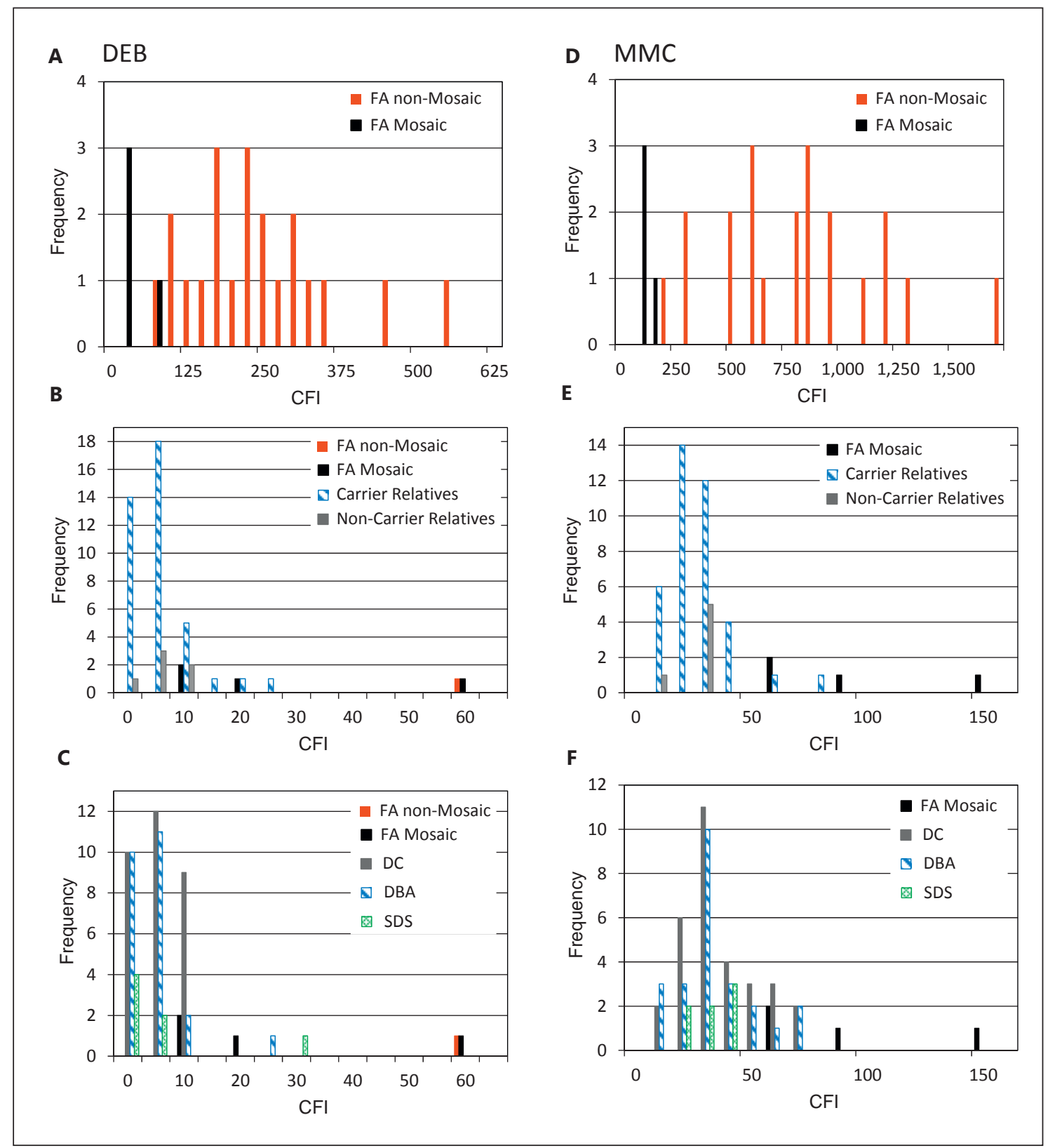

Fig. 6. Chromosome fragility index in cultures treated with DEB $(\mathbf{A}-\mathbf{C})$ and MMC (D-F). A, D Comparison of patients with nonmosaic FA with patients with mosaic FA. B, E Comparison of patients with mosaic FA with relatives. C, F Comparison of patients

tated. In this case, some of the patient's lymphocytes resemble those from heterozygotes. Although many mosaics have relatively normal peripheral blood counts, they remain at risk of aplastic anemia, MDS, or AML (which might develop from a latent non-mosaic stem cell), as well as solid tumors [Gregory et al., 2001; Alter et al., 2005; Sou- with mosaic FA with patients with other IBMFS. Symbols as in figure 2. One patient with FA with mosaicism has the same CFI as one without mosaicism, and the other 3 patients with mosaicism overlap with relatives and other IBMFS. lier et al., 2005]. It is also important to determine whether a hematologically normal sibling is truly an unaffected relative, or is an unrecognized patient with FA with mosaicism. Such an individual should not be a stem cell donor for his/her sibling with FA, and merits his/her own FAfocused genetic and clinical counseling and management. 
Several early studies determined that groups of carriers of FA had slightly more breakage than normal controls, but individual carrier status could not be predicted; the distinction of mosaics from carriers was not addressed at that time [Auerbach et al., 1981; Cervenka et al., 1981].

Several reports defined the parameters of mosaicism within their assays, and suggested that $10-25 \%$ of their series of patients with FA met their criteria. The diagnoses were based on the observation of 2 populations of lymphocytes, one of which was found to be resistant to DEB or MMC. The size of the resistant population in different studies varied, with low rates of breakage per cell, but with breaks per aberrant cell in the range seen in non-mosaic FA patients [Lo Ten Foe et al., 1997; Pearson et al., 2001; Alter et al., 2005; Soulier et al., 2005; Auerbach, 2009]. Most of those studies had small numbers of mosaic patients, and few studies proved mosaicism by documentation of high rates of chromosome breakage in skin fibroblasts, and/or demonstration of 1 mutated and 1 wild type FA allele in lymphocytes, with biallelic mutations in germline tissue such as fibroblasts [Lo Ten Foe et al., 1997; Alter et al., 2005]. Those studies also used a variety of clastogens, including $\mathrm{DEB}, \mathrm{MMC}$, and nitrogen mustard, at a variety of concentrations [Lo Ten Foe et al., 1997; Soulier et al., 2005; Auerbach, 2009].

We compared 2 clastogens in this study, DEB and $\mathrm{MMC}$, and confirmed that either clastogen could reliably identify most patients with FA. We sought an approach that would clearly distinguish between patients with FA with mosaicism, unaffected relatives, and patients with other IBMFS. To this end, we focused on the low range of percentage of aberrant cells, breaks per cell, and breaks per aberrant cell. As a group, relatives of patients with FA, both proven carriers and non-carriers (who were cytogenetically indistinguishable from each other), and patients with other IBMFS, had much less chromosomal breakage than patients with FA. The examination of individual data showed clearly that the patients with FA and mosaicism had lower values than the non-mosaics with all methods except breaks per aberrant cell, but there was overlap between the mosaics and the relatives or the other IBMFS with all methods, including breaks per aberrant cell. The latter is an interesting aside, which is that many apparently normal individuals do have a small number of cells with a substantial number of aberrant chromosomes with breaks and/or radials, such as described for CFI by Castella et al. [2011].

An important limitation of our study is that we studied only 4 mosaic patients, and thus the true range of chromosome breakage in those with mosaicism might be broader than that which we observed. A larger number of mosaics would strengthen our conclusions, if their breakage rates were similar to those in our study, or weaken them if the breakage rates were within the range of the non-mosaics. Our study also did not include concurrent analyses of normal individuals, because of the nature of the IBMFS cohort. A control sample was run with each experimental sample, but the control sample pool contained, in addition to non-FA individuals, individuals associated with FA, either carriers or non-carriers. We did not include these controls in our analyses in order to avoid confounding classification variables. We used concentrations of clastogens similar to the lower concentrations used by others, to avoid induction of breakage in normal individuals. In a conservative approach, we arbitrarily used 8 breaks or radials per cell as our upper limit, and counted anything beyond 8 as 8 , thus underestimating data in those patients with large numbers of radials, particularly using MMC.

A strength of our study is that it is the first to directly compare results in genetically classified patients with FA, mosaics (confirmed with skin fibroblast breakage, and FANC gene sequencing in blood and skin) and relatives, as well as genetically and clinically characterized patients with non-FA IBMFS, using syndrome-specific tests (e.g. red cell adenosine deaminase for DBA, telomere length for DC, and pancreatic enzymes for SDS) [Shimamura and Alter, 2010]. We simultaneously compared DEB and MMC in a single laboratory, and examined all proposed methods of analysis.

Despite all of the analytic methods evaluated, using both DEB and MMC in T lymphocyte assays, we found that mosaic patients with FA could not be unequivocally identified as such in the milieu of the other groups. Approximately $10 \%$ of relatives or patients with other IBMFS had aberrant cells, breaks per cell, or CFI numbers which were clearly below the levels of non-mosaic patients with FA, but above the lowest values in the mosaic patients with FA. In addition, about $10 \%$ of the relatives or patients with non-FA IBMFS had breaks per aberrant cells which overlapped with the patients with FA, including the mosaics. Thus, there did not appear to be a single, simple way to identify patients with FA and mosaicism, relatives of patients with $\mathrm{FA}$, or patients with non-FA IBMFS using clastogen-induced chromosome breakage in peripheral blood cells. Currently, chromosome breakage in skin fibroblasts and/or molecular assays of germline mutations provide definitive information. Perhaps a rapid marrow failure gene chip will provide a solution to this dilemma. Until then, clinical judgment remains of primary importance.
Fargo/Rochowski/Giri/Savage/Olson/ Alter 


\section{Acknowledgements}

We are grateful to all the patients who participate in the National Cancer Institute IBMFS cohort, to the physicians who referred the patients, and to our colleagues in the Clinical Genetics Branch of the National Cancer Institute and the subspecialty clinics at the National Institutes of Health for their evaluations of the patients. We thank Lisa Leathwood, RN; Ann Carr, MS, CGC;
Maureen Risch, RN and the other members of the IBMFS team at Westat, Inc. for their extensive efforts. This work was supported in part by the Intramural Program of the National Institutes of Health and the National Cancer Institute and by contracts N02-CP-11019, N02-CP-65504, and N02-CP-65501 as well as by a grant from the National Institutes of Health, Heart, Lung, and Blood Institute (NIH/NHLBI 5 P01 HL048546) to S.B.O.

\section{References}

-Alter BP, Joenje H, Oostra AB, Pals G: Fanconi anemia: adult head and neck cancer and hematopoietic mosaicism. Arch Otolaryngol Head Neck Surg 131:635-639 (2005).

- Alter BP, Rosenberg PS, Brody LC: Clinical and molecular features associated with biallelic mutations in FANCD1/BRCA2. J Med Genet 44:1-9 (2007).

-Alter BP, Giri N, Savage SA, Peters JA, Loud JT, et al: Malignancies and survival patterns in the National Cancer Institute inherited bone marrow failure syndromes cohort study. Br J Haematol 150:179-188 (2010).

Auerbach AD: Fanconi anemia and its diagnosis. Mutat Res 668:4-10 (2009).

- Auerbach AD, Wolman SR: Susceptibility of Fanconi's anaemia fibroblasts to chromosome damage by carcinogens. Nature 261:494-496 (1976).

Auerbach AD, Adler B, Chaganti RSK: Prenatal and postnatal diagnosis and carrier detection of Fanconi anemia by a cytogenetic method. Pediatrics 67:128-135 (1981).

- Berger R, Bernheim A, Gluckman E, Gisselbrecht $\mathrm{C}$ : In vitro effect of cyclophosphamide metabolites on chromosomes of Fanconi anaemia patients. Br J Haematol 45:565-568 (1980a).

-Berger R, Bernheim A, Le Coniat M, Vecchione D, Schaison G: Nitrogen mustard-induced chromosome breakage: a tool for Fanconi's anemia diagnosis. Cancer Genet Cytogenet 2: 269-274 (1980b).

Berwick M, Satagopan JM, Ben-Porat L, Carlson A, Mah K, et al: Genetic heterogeneity among Fanconi anemia heterozygotes and risk of cancer. Cancer Res 67:9591-9596 (2007).

Bogliolo M, Schuster B, Stoepker C, Derkunt B, Su $\mathrm{Y}$, et al: Mutations in ERCC4, encoding the DNA-repair endonuclease XPF, cause Fanconi anemia. Am J Hum Genet 92:800-806 (2013).
Castella M, Pujol R, Callen E, Ramirez MJ, Casado JA, et al: Chromosome fragility in patients with Fanconi anaemia: diagnostic implications and clinical impact. J Med Genet 48: 242-250 (2011).

Cervenka J, Arthur D, Yasis C: Mitomycin C test for diagnostic differentiation of idiopathic aplastic anemia and Fanconi anemia. Pediatrics 67:119-127 (1981).

Chen S, Parmigiani G: Meta-analysis of BRCA1 and $B R C A 2$ penetrance. J Clin Oncol 25: 1329-1333 (2007).

Deviren A, Yalman N, Hacihanefioglu S: Differential diagnosis of Fanconi anemia by nitrogen mustard and diepoxybutane. Ann Hematol 82:223-227 (2003).

Dokal I, Luzzatto L: Dyskeratosis congenita is a chromosomal instability disorder. Leuk Lymphoma 15:1-7 (1994).

Gregory JJ Jr, Wagner JE, Verlander PC, Levran $\mathrm{O}$, Batish SD, et al: Somatic mosaicism in Fanconi anemia: evidence of genotypic reversion in lymphohematopoietic stem cells. Proc Natl Acad Sci USA 98:2532-2537 (2001).

Gross M, Hanenberg H, Lobitz S, Friedl R, Herterich $\mathrm{S}$, et al: Reverse mosaicism in Fanconi anemia: natural gene therapy via molecular self-correction. Cytogenet Genome Res 98: 126-135 (2002).

Higurashi M, Conen PE: In vitro chromosomal radiosensitivity in Fanconi's anemia. Blood 38:336-342 (1971).

Kwee ML, Poll EHA, Van de Kamp JJP, de Koning $\mathrm{H}$, Eriksson AW, Joenje H: Unusual response to bifunctional alkylating agents in a case of Fanconi anaemia. Hum Genet 64:384-387 (1983).
Lo Ten Foe JR, Kwee ML, Rooimans MA, Oostra $\mathrm{AB}$, Veerman AJP, et al: Somatic mosaicism in Fanconi anemia: molecular basis and clinical significance. Eur J Hum Genet 5:137-148 (1997).

Mankad A, Taniguchi T, Cox B, Akkari Y, Rathbun RK, et al: Natural gene therapy in monozygotic twins with Fanconi anemia. Blood 107:3084-3090 (2006).

Pearson T, Jansen S, Havenga C, Stones DK, Joubert G: Fanconi anemia: a statistical evaluation of cytogenetic results obtained from South African families. Cancer Genet Cytogenet 126:52-55 (2001).

Rosenberg PS, Tamary H, Alter BP: How high are carrier frequencies of rare recessive syndromes? Contemporary estimates for Fanconi anemia in the United States and Israel. Am J Med Genet A 155A:1877-1883 (2011).

Sasaki MS, Tonomura A: A high susceptibility of Fanconi's anemia to chromosome breakage by DNA cross-linking agents. Cancer Res 33: 1829-1836 (1973).

-Schroeder TM, Anschütz F, Knopp A: Spontane Chromosomenaberrationen bei familiärer Panmyelopathie. Humangenetik 1:194-196 (1964).

Schuler D, Kiss A, Fábián F: Chromosomal peculiarities and 'in vitro' examinations in Fanconi's anaemia. Humangenetik 7:314-322 (1969).

-Shimamura A, Alter BP: Pathophysiology and management of inherited bone marrow failure syndromes. Blood Rev 24:101-122 (2010).

Soulier J, Leblanc T, Larghero J, Dastot H, Shimamura A, et al: Detection of somatic mosaicism and classification of Fanconi anemia patients by analysis of the FA/BRCA pathway. Blood 105:1329-1336 (2005). 